\title{
Is science as global as we think?
}

\author{
Bob B.M. Wong ${ }^{1,2}$ and Hanna Kokko ${ }^{3}$
}

${ }^{1}$ School of Botany and Zoology, The Australian National University, Canberra, ACT 0200, Australia

${ }^{2}$ Boston University Marine Program, 7 MBL Street, Woods Hole, MA 02543, USA

${ }^{3}$ Laboratory of Ecological and Evolutionary Dynamics, Department of Biological and Environmental Science, University of Helsinki, P.O. Box 65 (Viikinkaari 1), FIN-00014 Helsinki, Finland

Corresponding author: Kokko, H. (hanna.kokko@helsinki.fi) 
A cornerstone of scientific practice is the perceived freedom with which knowledge is disseminated. But does the scientific community make good use of all that is available? Researchers have, on occasion, been accused of preferentially citing the work of colleagues close to home while ignoring those from other parts of the world $[1,2]$. Such practices, if true, raises serious concerns about the free flow of scientific information [1]. Recently, Leimu and Koricheva [3] showed that ecological papers written by US authors attracted significantly more citations than did papers by European authors. They suggested that such differences could, potentially, be due to parochial citation practices; papers by US based authors could receive more citations if they are attracting, not only their fair share of European citations, but also a disproportionate share of citations from the US ([3], see also [1, 4]). In one journal (Oecologia), such a pattern was indeed found [5]. Here, we test if such a geographic citation bias extends to other journals and different types of papers, and if biases diminish over time as important results get more widely known.

For analysis, we compared publications from two major geographic regions: Europe (Norway, Switzerland, and European Union countries) and North America (USA and Canada). Both focal (cited) papers and citing papers were only included in the analysis if authors' affiliations were all inside one of these regions. Citing articles were included irrespective of the journal they were published in, and were found using the "citation search' service of Web of Science (INC information), up to publication year 2003. Self- 
citations (citing papers that had authors in common with the cited paper) were excluded from analysis.

Parochial citing practices could arise through differences in the readership of American versus European journals (i.e. if Americans do not read European journals and vice versa, [1]). But if papers published in the same journal are cited differently depending on the continent the citing author resides, evidence for parochial practices becomes much stronger. We therefore tracked the fate of papers by North American- and Europeanbased authors publishing in the same journal, focusing on recent (2000) and relatively older (1997) papers appearing in Ecology (published in the US; $n=60$ in 1997, 75 in 2000) and Journal of Animal Ecology (published in Europe; $n=61$ in 1997, 73 in 2000). We also included a random sample of 24 papers published in Nature in 1997. Our results indicate geographic "over-citing" on both sides of the Atlantic; papers were cited relatively more if the citing and cited authors resided in the same geographic region (fig. $1 \mathrm{a}-\mathrm{c})$.

While evidence for local over-citing is strong, our results do not reveal if researchers in one region are more to blame than the other. This is because it is impossible to judge what an unbiased rate of citation for European or North American papers should be [5]. For example, if North Americans truly produced better science, the signature of overciting in that part of the world would be justified, and the European bias would then be a sign of parochial practices (and vice versa). Notwithstanding such a possibility, however, 
the strength of geographic over-citing still raises concerns that both sides of the Atlantic could be engaging in parochial citation practices.

It is particularly alarming that differences do not diminish for older papers which should have had more time to become known by both Americans and Europeans regardless of who originally published the work. Of course, for empirical studies, differences could, to some extent, reflect acceptable patterns of local citing because they concern local natural history. Yet, theoretical papers (defined as no original data presented in the paper) published between 1984-2002 showed similar fates (fig. 1d; $n=59$ for Ecology, $n=42$ for J.Anim.Ecol.), although in theoretical papers published in Journal of Animal Ecology significant geographic over-citing is, intriguingly, replaced by papers of European origin being cited more by researchers on both sides of the Atlantic.

Our results, together with those reported in [5], suggest that both the empirical and theoretical advancement of ecology are significantly differentiated between Europe and North America. To a certain degree, this could reflect relatively neutral factors, such as differences in the topics ecologists from different regions choose to specialize in. If, however, researchers are overlooking or ignoring the work of colleagues from elsewhere [1], fair recognition of individual scientists' efforts is far from guaranteed, and the advancement of science itself is considerably hampered.

Acknowledgements 
We thank Michael Jennions for statistical advice. An Australian Postgraduate Award and a Sir Keith Murdoch Fellowship (to BBMW) and the Academy of Finland (to HK) provided funding.

\section{Reference}

1 Møller, A.P. (1990) National citatios. Nature 348, 480.

2 Wardle, D.A. (1995) Journal citation impact factors and parochial citation practices. Bull. Ecol. Soc. Am. 76, 102-104.

3 Leimu, R. and Koricheva, J. (2005) What determines the citation frequency of ecological papers? Trends Ecol. Evol. 20, 28-32.

4 Paris, G. et al. (1998) Region-based citation bias in science. Nature 396, 210.

5 Leimu, R. \& Koricheva, J. (2005) Does scientific collaboration increase the impact of ecological articles? Bioscience 55, 438-443.

\section{Figure Legends}

Fig. 1 Observed citation patterns in three journals (Ecology: squares, J.Anim.Ecol.: dots, triangles: Nature). Mean \pm s.e. for cumulative citation sums ( $\Sigma E U R, \Sigma N A)$ to each focal paper are given (European authors: open symbols, North American authors: filled symbols). P-values indicate geographic 
overciting within a journal, derived from one-tailed rank sum tests for a difference in the angle through origin. The citation sums to each focal article provide one data point for the angle. If the papers differ in the total citation numbers, the pattern can arguably be explained by superior quality of papers originating from a given geographical area, but there are no consistent differences of this type between articles from Europe and North America. Instead, we found systematic differences in the angle through origin, which indicates geographical overciting. 


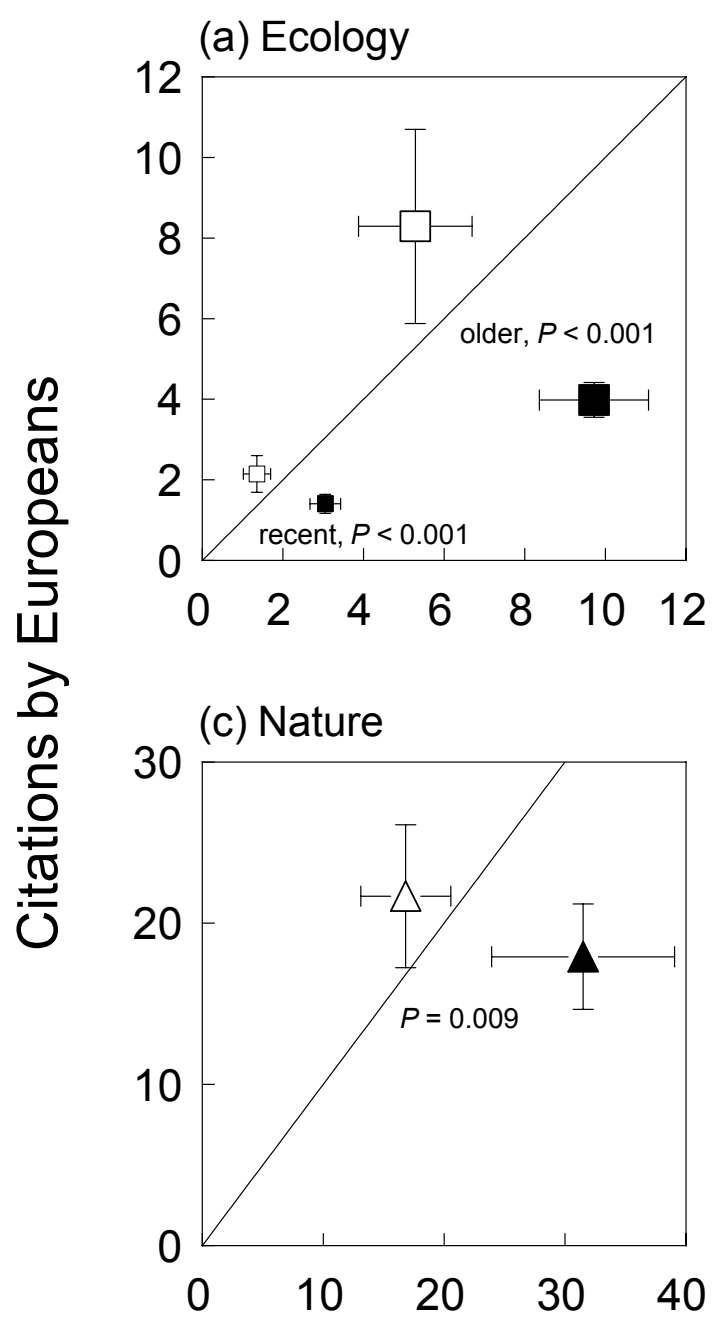

(b) J. Anim. Ecol.
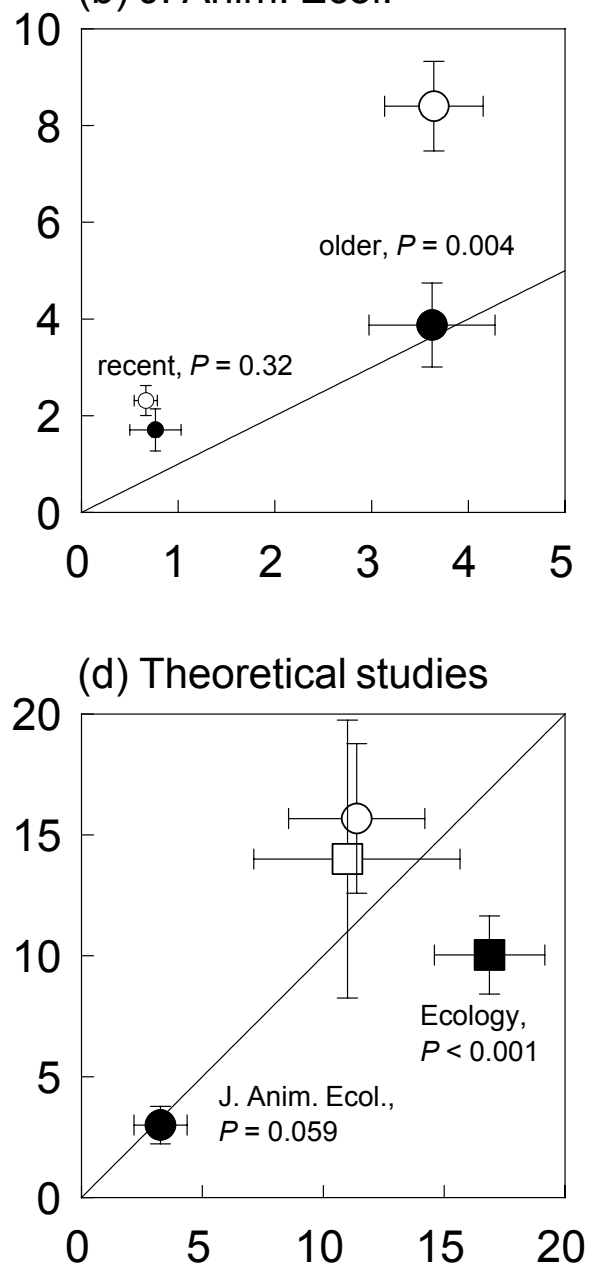

Citations by North Americans 